\title{
Homology-based prediction of interactions between proteins using Averaged One-Dependence Estimators
}

\author{
Yoichi Murakami ${ }^{1,2^{*}}$ and Kenji Mizuguchi ${ }^{*^{*}}$
}

\begin{abstract}
Background: Identification of protein-protein interactions (PPIs) is essential for a better understanding of biological processes, pathways and functions. However, experimental identification of the complete set of PPIs in a cell/organism ("an interactome") is still a difficult task. To circumvent limitations of current high-throughput experimental techniques, it is necessary to develop high-performance computational methods for predicting PPIs.

Results: In this article, we propose a new computational method to predict interaction between a given pair of protein sequences using features derived from known homologous PPIs. The proposed method is capable of predicting interaction between two proteins (of unknown structure) using Averaged One-Dependence Estimators (AODE) and three features calculated for the protein pair: (a) sequence similarities to a known interacting protein pair $\left(F_{\text {Seq }}\right)$, (b) statistical propensities of domain pairs observed in interacting proteins $\left(F_{\text {Dom }}\right)$ and $(c)$ a sum of edge weights along the shortest path between homologous proteins in a PPI network ( $\left.F_{N e t}\right)$. Feature vectors were defined to lie in a half-space of the symmetrical high-dimensional feature space to make them independent of the protein order. The predictability of the method was assessed by a 10 -fold cross validation on a recently created human PPI dataset with randomly sampled negative data, and the best model achieved an Area Under the Curve of $0.79\left(\mathrm{pAUC}_{0.5 \%}=0.16\right)$. In addition, the AODE trained on all three features (named PSOPIA) showed better prediction performance on a separate independent data set than a recently reported homology-based method.
\end{abstract}

Conclusions: Our results suggest that $\mathrm{F}_{\mathrm{Net}}$ a feature representing proximity in a known PPI network between two proteins that are homologous to a target protein pair, contributes to the prediction of whether the target proteins interact or not. PSOPIA will help identify novel PPIs and estimate complete PPI networks. The method proposed in this article is freely available on the web at http://mizuguchilab.org/PSOPIA.

Keywords: Prediction of protein-protein interactions, Homology, Machine learning, Averaged One-Dependence Estimators (AODE)

\section{Background}

Many biological processes and pathways are mediated by protein-protein interactions (PPIs). Identification of individual PPIs and the whole set of them in a cell/organism ("an interactome") is, therefore, essential for a better understanding of biological functions of proteins in living cells and elucidating biochemical pathways. Various high-throughput experimental techniques, such as yeast two-hybrid assays

\footnotetext{
*Correspondence: yoichi@ecei.tohoku.ac.jp; kenji@nibio.go.jp

'Bioinformatics Project, National Institute of Biomedical Innovation, 7-6-8 Saito-Asagi, Ibaraki, Osaka 567-0085, Japan

${ }^{2}$ Graduate School of Information Sciences, Tohoku University, 6-3-09

Aramakiaza-aoba, Aoba-ku, Sendai, Miyagi 980-8579, Japan
}

and methods based on mass spectrometry, have been used to discover a large number of PPIs in several organisms. Although the amount of interaction data in public PPI databases continues to rise, many of them represent an incomplete interactome, because the available experimental techniques are expensive and can typically identify only a small part of the set of PPIs in specific organisms [1,2].

To circumvent such limitations of the experimental techniques, a number of computational methods have been developed for predicting PPIs based on prior knowledge obtained from known interacting protein sequences and using machine-learning (ML) techniques [3-14]. Efforts have been made to develop methods based only on 
information about amino acid sequences, for example, by using the number of amino acid triplets in each sequence $[6,10,13]$, a product of signatures defined as a set of subsequences [7], auto-correlation values of seven different physicochemical scales $[11,15]$ and normalized counts of single or pairs of consecutive amino acid residues [12]. These purely sequence-based approaches have reported prediction accuracies of $70-84 \%$ on a human data set and about $70 \%$ on a yeast data set. Furthermore, information about protein domains has been incorporated in several other methods [16,17]. Although it has been shown to be an informative feature for predicting PPIs [14], methods utilizing domain information alone are not applicable to proteins without domain assignments.

Identifying proteins homologous to a newly determined protein is often attempted to infer the biological functions of the new protein of unknown function, because homologues tend to have similar functions as well as similar three-dimensional structures. This deductive inference has been applied to the identification of PPIs, on the assumption that homologous proteins share similar interaction patterns as well as similar functions [18]. A pair of interacting proteins in one species and their respective orthologs in another species, which are also known to interact with each other, have been traditionally defined as interaction-orthologs (interologs) [19,20]. However, this idea can be extended to interaction-homologs, because orthologs and paralogs are not always clearly distinguished [18,21].

There have been several computational studies about interologs. For example, Yu et al. found that PPIs can be transferred when two pairs of proteins have the geometric mean of the sequence identities $>80 \%$ or the e-values $<10^{-70}$ [20]. Wiles et al. predicted PPIs from known interactions in five species and developed InterologFinder, a web server to search for information about predicted as well as experimentally determined PPIs for given proteins of interest [22]. Chen et al. developed PPISearch, a web server to search for homologous PPIs given a single protein pair of interest against an integrated database of PPIs in 576 species [18]. Gallone et al. developed a Perl module to search for putative PPIs and prioritize them based on interologs [23]. Garcia et al. developed BIPS, a web server to predict PPIs based on information about known PPIs in multiple species and additional information about domain interactions and GO annotations. It uses BIANA, an integrated database of PPIs from several repositories [21,24]. In these prediction approaches, collecting as many PPIs as possible in multiple species is an important factor for the reliability of the predicted interactions.

Furthermore, developing a confidence score for PPIs is also key to improving the reliability of the prediction. Most of the previously reported methods used a simple joint sequence identity or e-value for two pairs of interacting proteins $[18,20,21]$, whereas one unified score based on the level of homology, conservation of the interactions across multiple species and the number of supporting experimental types was proposed [22]. These methods are largely dependent on the existence of orthologous or homologous PPIs, i.e., it would be very difficult to detect a novel PPI with no interlogs in an integrated database.

To improve the discrimination power of the homologybased PPI prediction, we here apply Averaged OneDependence Estimators (AODE; [25]) to this problem. The AODE is an ML algorithm, a variant of the Naive Bayes classifier (NBC) and it weakens NBC's independence assumption by allowing a one-dependence. So far, the AODE has been used to combine the outputs of several protein interaction prediction methods; it has been shown to be useful for extracting distinctive information from large imbalanced datasets and it can also be retrained easily and efficiently [26]. Furthermore, it has been reported to be more accurate than NBC, and it can efficiently process a large number of training feature vectors in a high dimensional space without increasing the computational cost significantly [25,27]. In addition, the AODE does not need to select a model and to optimize any parameters. These strengths, therefore, allowed us to train the AODE on massive PPI data collected from several repositories without incurring a large computational cost.

In this study, the AODE is trained using three features: (a) sequence similarities to known interacting proteins $\left(\mathrm{F}_{\mathrm{Seq}}\right)$, (b) statistical propensities of domain pairs observed in interacting proteins $\left(\mathrm{F}_{\text {Dom }}\right)$ and $(\mathrm{c})$ a sum of edge weights along the shortest path between homologous proteins in a PPI network $\left(\mathrm{F}_{\mathrm{Net}}\right)$. The idea of feature (c) is based on the hypothesis that a target protein pair would have more potential to interact if their homologous proteins exist in proximity of each other in a known PPI network. Such a proximal pair, even if not known to interact directly, may form a complex with other proximal proteins or reside in common subcellular locations, thereby increasing the chances of their homologues interacting directly. In a previous study, the topology of a PPI network has been used to predict interactions missing in the network (i.e., those not detected by largescale experiments), by searching for defective cliques (with a few missing edges) in the PPI network graph [28]. However, this approach can be applied only to proteins with at least one experimentally defined interaction. In addition, the computational cost of this method has been reported to be expensive. Our method, in contrast, searches for a pair of sequences in the graph homologous to the query proteins, which may be unannotated and with no known interactions. Then, a sum of edge 
weights along the shortest path between them is computed and trained with other features, thus dramatically reducing the computational cost. We demonstrate high predictive performance of the AODE on a recently created human PPI data set with randomly sampled negative data [29], which had been used for benchmarking previously reported sequence-based methods.

\section{Methods}

In this section, we first introduce the data set used for training and testing, and describe three features calculated for a pair of proteins. Next, we describe how to construct a feature vector, dealing with symmetry in the protein order. Then, we describe the AODE for probabilistic classification of protein pairs into interacting (positive) or non-interacting (negative) classes, and introduce prediction accuracy measures to assess prediction models developed and the validation method.

\section{Preparation of a PPI data set}

To train and evaluate AODEs for predicting PPIs, we used two different datasets:

(1) Dset1 is a recently created non-redundant human PPI data set (ensuring $\leq 40 \%$ pairwise sequence identity and protein sequence length of $>50$ amino acids) obtained from the Human Protein Reference Database (HPRD; release 7; [30]), created by [29]. This data set was divided into three independent sets, each of which contained about 2,000 proteins with about 5,000 positive pairs and 2,000,000 negative pairs, i.e., 400 times larger number of non-interacting protein pairs, generated by randomly paring proteins that appeared in the positive pairs and removing real positive pairs. This is a highly imbalanced data set and the classification categories are unequally represented. Park and Marcotte used these subsets to benchmark four different sequence-based PPI prediction methods [29,31] (see Additional file 1: Table S1).

(2) Dset2 was constructed to compare prediction performance of the AODE trained on Dset1 with BIPS, a recently developed homology-based prediction server [21]. First, a set of human physical PPIs was obtained from the BioGrid dataset (release 3.2.95, December 2012). Then, from this dataset, we removed PPIs found in the previous BioGrid dataset (release 3.1.93, on October, 2012) compiled after BIPS was released, ensuring that Dset2 includes only recently discovered PPIs. In addition, we used only a set of interacting proteins, each of which was annotated in UniProt [32]. This procedure left a set of 4.430 PPIs. Finally, negative PPI pairs 400 times larger in number than the positives ones were generated in a manner similar to that of Dset1.
Homology-based features for a pair of proteins

The following three features were calculated for a pair of proteins $\left(S_{A}, S_{B}\right)$;

\section{(a) Sequence similarities to known interacting} proteins $\left(\mathbf{F}_{\text {Seq }}\right)$ : Known interacting pairs with sequence similarity to a target pair $\left(S_{A}, S_{B}\right)$ were searched by running BLAST (version 2.2.25+; [33]) against the database created from the sequences in Dset1, with an e-value cutoff of $\leq 10^{2}$. (The high e-value cutoff was chosen to allow for partial matches). Then, of these pairs, the interacting pair $\left(T_{A}, T_{B}\right)$ with the smallest value of $\sqrt{ }\left(e-v_{\text {alue }}^{2}+\right.$ $e$-value $\left.e_{B}^{2}\right)$ was selected, where $e$-value $e_{x}$ is the BLAST e-value between $S_{x}$ and $T_{x}$ and $x$ is either A or B. The minimum coverage (mincov) for $S_{x}$ and $T_{x}$ was also calculated as the number of positive matches (i.e., alignment positions with a positive BLOSUM62 score [34]) divided by the length of the longer sequence. These two BLAST e-values and two minimum coverage values, $\left(e\right.$-value ${ }_{A}$, mincov $\left._{A}\right)$ for $S_{A}$ and $\left(e-\right.$ value $_{B}$, mincov $\left._{B}\right)$ for $S_{B}$, were used as features for training (Figure 1-a). If no known homologous interacting pair was found, an e-value of $10^{2}$ and a mincov of 0 were assigned to $\mathrm{F}_{\text {Seq. }}$.

(b) Statistical propensities of domain pairs observed in interacting proteins $\left(\mathbf{F}_{\text {Dom }}\right)$ : Each sequence in Dset1 was scanned against Pfam-A (release 25.0; Pfam-A.hmm; [35]), and the number of Pfam domain pairs $\left(d_{A}, d_{B}\right)$ that appeared in either positive or negative pairs was counted. Knowledge-based interaction propensities for Pfam domain pairs were calculated as:

$\operatorname{propensity}\left(d_{A}, d_{B}\right)=\log \left(\frac{F\left(+, d_{A}, d_{B} / \sum_{x, y \in D} F\left(+, d_{x}, d_{y}\right)\right.}{F\left(-, d_{A}, d_{B} / \sum_{x, y \in D} F\left(-, d_{x}, d_{y}\right)\right.}\right)$

where $F\left(c, d_{A}, d_{B}\right)$ is the frequency of a domain pair $\left(d_{A}, d_{B}\right)$ observed in protein pairs belonging to class $c$ (+; positive, -; negative), and $D$ is a set of all Pfam domains observed in Dset1. For each target protein pair, a sum of the interaction propensities for all possible Pfam domain pairs was obtained and divided by the number of the domain pairs. If no Pfam domain was found in $S_{A}$ and/or $S_{B}$, an $\mathrm{F}_{\text {Dom }}$ value of 0 was given to the target pair (Figure 1-b).

\section{(c) A sum of edge weights along the shortest path between homologous proteins in the PPI} network $\left(\mathbf{F}_{\mathrm{Net}}\right)$ : BLAST hits (with an e-value cutoff $\leq 10^{-3}$ ) for each sequence in a target pair $\left(S_{A}, S_{B}\right)$ were collected from the database created from Dset1. Then, for each possible pair of hits $\left(p_{A}, p_{B}\right)$, where $p_{A}$ and $p_{B}$ were among the hits for $S_{A}$ and $S_{B}$, respectively, a sum of edge weights along the 
(a) Sequence similarities to a known interacting protein pair

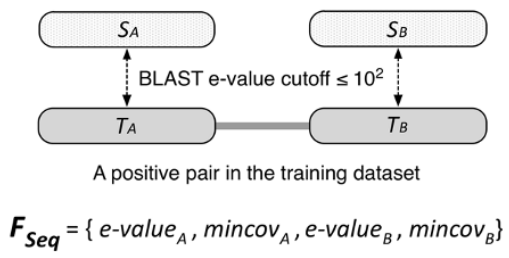

(b) Statistical propensities of domain pairs observed in a protein pair

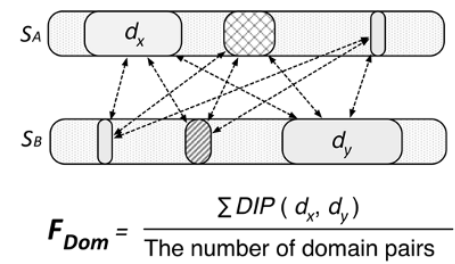

(c) A sum of edge weights along the shotest path between homologous proteins in PPI network

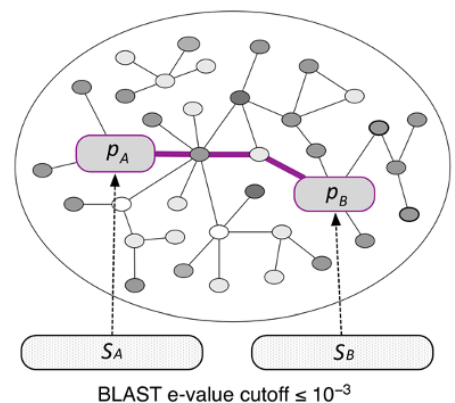

PPI network (a weight of an edge $=1.0$ )

protein

- interaction

$\boldsymbol{F}_{\boldsymbol{N e t}}=\mathrm{SPW}$ between two similar proteins $\left(p_{A}, p_{B}\right)$

Figure 1 Three homology-based features used for training AODEs. (a) A feature set of sequence similarities to known interacting proteins $\left(F_{\text {Seq }}=\left\{e-\right.\right.$ value $_{A}$, mincov $_{A}, e-$-value $_{B}$, mincov $\left.\left._{B}\right\}\right)$. For a target pair $\left(S_{A}, S_{B}\right)$, the interacting pair $\left(T_{A}, T_{B}\right)$ with the smallest value of $\sqrt{ }\left(e-v a l u e_{A}^{2}+e-v a l u e e_{B}^{2}\right)$, where $e$-value $x$ is a BLAST e-value between $S_{x}$ and $T_{x}$ and $x$ is either $A$ or $B$, is selected, and then the minimum coverage (mincov) for $S_{x}$ and $T_{x}$ is calculated as (the number of positive matches)/(the length of the longer sequence). If no known homologous interacting pair is found, an $e$-value of $10^{2}$ and a mincov of 0 are assigned to $F_{\text {seq. }}$ (b) Statistical propensities of domain pairs observed in interacting proteins ( $F_{\text {Dom }}$ ). A sum of the interaction propensities for all possible Pfam domain pairs $\left(d_{A}, d_{B}\right)$ appeared in $S_{X}$ and $T_{X}$ is calculated (see more details in the text). If not Pfam domain is found, an $\mathrm{F}_{\text {Dom }}$ value of 0 is given to the target pair. (c) A sum of edge weights along the shortest path between homologous proteins $\left(P_{A}, P_{B}\right)$ in the PPI network $\left(F_{\text {Net }}\right)$. In this study, we set the default edge weight to be 1.0. If no path is found, an $F_{\text {Net }}$ of -1 is given to the target pair.

shortest path (the shortest path weight; SPW) was calculated. In this study, we set the default edge weight to be 1.0. The shortest path between $p_{A}$ and $p_{B}$ was calculated using Dijkstra's shortest path algorithm implemented in the Boost::Graph perl module (version 1.4; downloaded from http://search. cpan.org/ dburdick/Boost-Graph/), which is a perl interface to the Boost-Graph $\mathrm{C}++$ libraries (release 1.47.0; downloaded from http://www.boost.org/). The lowest SPW was used as a feature for training. If no SPW was defined for any of the pairs $\left(p_{A}, p_{B}\right)$, an $\mathrm{F}_{\text {Net }}$ value of -1 was given to the target pair (Figure 1-c).

\section{Constructing a feature vector}

For each target protein pair, three sequence features described above were computed and converted into a feature vector $(\mathrm{FV})=\left\{\mathrm{F}_{\text {Seq }}, \mathrm{F}_{\mathrm{Dom}}, \mathrm{F}_{\mathrm{Net}}\right\}$. However, at least two feature vectors can be constructed for $\mathrm{F}_{\mathrm{Seq}}$, depending on the order of the two protein, i.e., $\mathrm{F}_{\mathrm{Seq}}=\left\{e-v^{\mathrm{alu}} \mathrm{e}_{A}\right.$, mincov $_{A}, e-$ value $_{B}$, mincov $\left._{B}\right\}$ and $\mathrm{F}_{\mathrm{Seq}}{ }^{\prime}=\left\{e-\right.$ value $_{B}$, min$\operatorname{cov}_{B}, e-$ value $_{A}$, mincov $\left._{A}\right\}$, and in general, $\mathrm{F}_{\text {Seq }}{ }^{\prime} \neq \mathrm{F}_{\text {Seq. }}$. To define a FV uniquely, we first chose an arbitrary pair of proteins whose $\mathrm{F}_{\text {Seq }}$ and $\mathrm{F}_{\text {Seq }}$ ' values corresponded to points $X_{1}$ and $X_{2}$ in the feature space (Figure 2). These points are symmetrically arranged in the four-dimensional feature space separated by a hyperplane. Of the two possible values for any protein pair, we decided to take the one corresponding to a point on the same side of the hyperplane as $X_{1}$ and denoted this value as $\mathrm{F}_{\text {Seq }}$. More precisely, for a given pair of proteins, $\mathrm{F}_{\mathrm{Seq}}$ " was defined by the point $P_{1}$ that had $\cos \theta=r n \cdot V /|r n||V|>$ 0 , where $r n$ is a reference normal vector from the midpoint $(r p)$ between $X_{1}$ and $X_{2}$ to $X_{1}, V$ is a vector from $r p$ to $P_{1}, r n \cdot V$ is the inner product of $r n$ and $V$ and $|r n|$ and $|V|$ are the lengths of $r n$ and $V$, respectively. If $\cos$ $\theta=0$, one of the two possibilities was arbitrarily selected as $\mathrm{F}_{\mathrm{Seq}}$ ". Finally, a unique $\mathrm{FV}$ was constructed as $\left\{\mathrm{F}_{\mathrm{Seq}} ", \mathrm{~F}_{\mathrm{Dom}}, \mathrm{F}_{\mathrm{Net}}\right\}$.

After the construction of $\mathrm{FVs}$, feature values for $i$-th feature of the FVs used for training were discretized using the entropy-based discretization method [36]. The optimized intervals (split points), the number of which varied with each feature, were then applied to the construction of FVs for testing. 


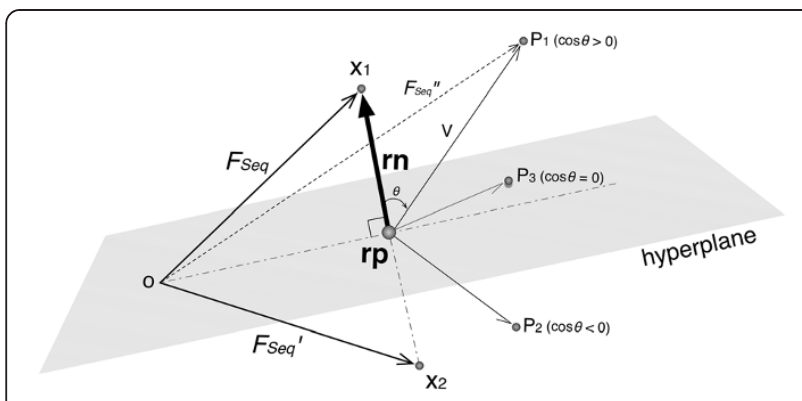

Figure 2 Selection of feature vectors in the half-space defined by $\mathbf{X} 1$. To define a FV uniquely, an arbitrary pair of proteins whose $F_{\text {Seq }}$ and $F_{\text {Seq }}$ values corresponded to points $X_{1}$ and $X_{2}$ in the highdimensional feature space is first chosen. For a given pair of proteins,

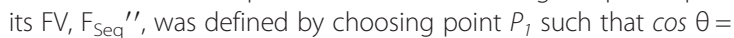
$r n \cdot V /|r n| \mid V>0$, where $r n$ is a reference normal vector from the midpoint between $X_{1}$ and $X_{2}(r p)$ to $X_{1}, V$ is a vector from $r p$ to $P_{1}$, $r n \cdot V$ is the inner product of $r n$ and $V$ and $|r n|$ and $\mid V$ are the length of $r n$ and $V$, respectively. If $\cos \theta=0$, one of the two possibilities was arbitrarily selected.

\section{Averaged One-Dependence Estimator (AODE)}

The AODE weakens NBC's independence assumption by allowing a one-dependence, i.e., allowing each feature to depend on another single feature (Figure 3), and it averages the predictions of all one-dependence estimators (ODEs) in each class [25]. The AODE estimates the probability of the positive class $(+)$ given a specified set of features $\left\{f_{1}, f_{2}, \ldots, f_{n}\right\}$, and is calculated as:

$$
\hat{P}\left(+\mid f_{1}, f_{2}, \ldots, f_{n}\right)=\frac{\sum_{i=1}^{n} \hat{P}\left(+, f_{i}\right) \prod_{j=1 \wedge i \neq j}^{n} \hat{P}\left(f_{j} \mid+, f_{i}\right)}{\sum_{c \in\{+,-\}} \sum_{i=1}^{n} \hat{P}\left(c, f_{i}\right) \prod_{j=1 \wedge i \neq}^{n} \hat{P}\left(f_{j} \mid c, f_{i}\right)}
$$

Here the base probabilities $\hat{P}\left(c, f_{i}\right)$ and $\hat{P}\left(c, f_{i}, f_{j}\right)$ were estimated with the Laplace smoothing as:

$$
\begin{aligned}
& \hat{P}(c, f)=\frac{F\left(c, f_{i}\right)+1}{m_{i}+k v_{i}} \\
& \hat{P}\left(c, f_{i}, f_{j}\right)=\frac{F\left(c, f_{i}, f_{j}\right)+1}{m_{i j}+k v_{i} v_{j}}
\end{aligned}
$$

where $F(\cdot)$ is the frequency with which a combination of terms appeared in the training FVs, $m_{i}$ is the number of training FVs for which the $i$-th feature were known, $m_{i j}$ is the number of training FVs for which the $i$-th and $j$-th features were known, $c$ is a class label out of a total of $k(=2)$ classes, and $v_{i}$ and $v_{j}$ are the number of discrete partitions for the $i$-th and $j$-th features, respectively. Then, the conditional probability $\hat{P}\left(f_{j} \mid c, f\right)$ was estimated as:

$$
\hat{P}\left(f_{j} \mid c, f_{i}\right)=\frac{\hat{P}\left(c, f_{i}, f_{j}\right)}{\hat{P}\left(c, f_{i}\right)}
$$

A probabilistic graphical model of the AODE modeled in this study is shown in Figure 3.

If the probability is greater than or equal to a threshold, the target pair is predicted to be interacting, otherwise noninteracting. A schematic diagram of the prediction procedure is summarized in Figure 4.

\section{Evaluation measures and validation}

Performances of AODEs were estimated by the Area Under the Curve (AUC), which gives an $\mathrm{AUC}=1.0$ for a perfect model and gives an $\mathrm{AUC}=0.5$ for a random model for which a Receiver Operating Characteristic (ROC) curve is drawn as a diagonal line. A ROC curve is most often used for model comparison and is represented by plotting sensitivity (true positive rate; TPR, or recall) against 1.0 - specificity (false positive rate; FPR). Sensitivity (recall) measures the proportion of the known
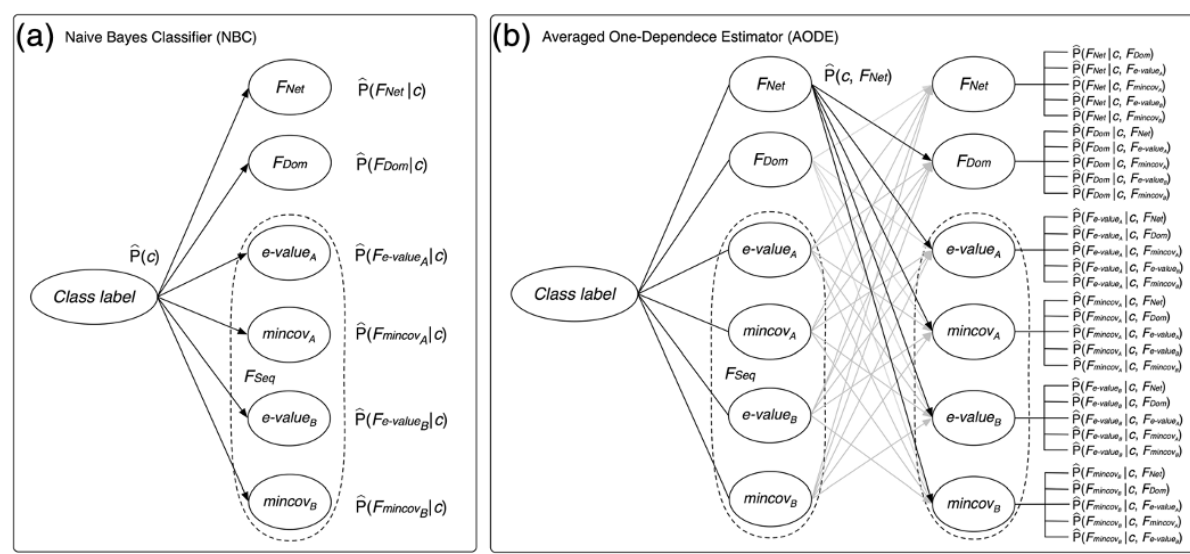

Figure 3 A probabilistic graphical model of the AODE. The AODE weakens the NBC's independence assumption, of which the dependence structure is shown in (a), by allowing a one-dependence as shown in (b). $\hat{P}(c)$ is the probability of each class label $c \in\left\{\right.$ interaction or non-interaction\}, $\hat{P}\left(f_{i} \mid c\right)$, such as $\hat{P}\left(F_{\text {Net }} \mid c\right)$, is the conditional probability of the $i$-th feature $\left(f_{i}\right)$ given $c, \hat{P}\left(c, f_{i}\right)$, such as $\hat{P}\left(c, F_{\text {Net }}\right)$, is the joint probability of $c$ and $f_{j}$ and also $\hat{P}\left(f_{j} \mid c, f_{i}\right)$, such as $\hat{P}\left(F_{\text {Net }} \mid c, F_{\text {Dom }}\right)$, is the conditional probability of the $j$-th feature $\left(f_{j}\right)$ given $c$ and $f_{i}$ 


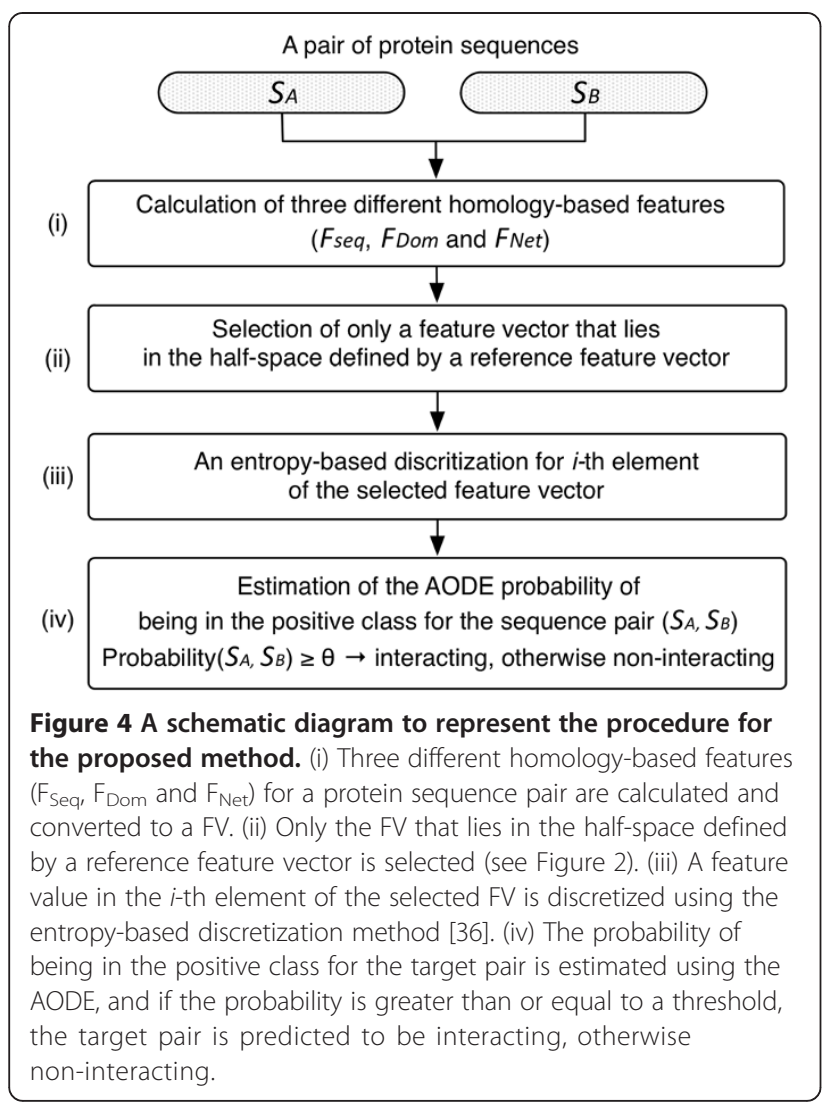

positive pairs that are correctly predicted as interacting and is defined as TP/(TP + FN), and specificity measures the proportion of the known negative pairs that are correctly predicted as non-interacting and is defined as TN/ $(\mathrm{TN}+\mathrm{FP})$, where TP is the number of true positives (i.e., known positive pairs correctly predicted as interacting), FP is the number of false positives (i.e., known negative pairs incorrectly predicted as interacting), $\mathrm{TN}$ is the number of true negatives (i.e., known negative pairs correctly predicted as non-interacting), and FN is the number of false negatives (i.e., known positive pair incorrectly predicted as non-interacting). The AUC is known to be insensitive to imbalanced data [37] and it would be a reliable measure for the prediction performance. In addition, performances of AODEs were also estimated by a normalized partial AUC up to the FPR $\leq x \%$ (pAUC $x \%$ ), following [6] and [14]. We set $x$ to be 0.5 . A prediction model with a high pAUC can predict more true positives with few FPs, so such a model is known to be most useful for users to identify PPIs from the top-ranked predictions [6].

Furthermore, we used two other common measures, MCC (Mathew's correlation coefficient; [38]) and the Fmeasure [39]. MCC indicates the degree of the correlation between the actual and predicted classes of the protein pair, and its values range between 1 where all the predictions are correct, and -1 where none are correct. MCC is defined as $(\mathrm{TP} \times \mathrm{TN}-\mathrm{FP} \times \mathrm{FN}) / \sqrt{ }(\mathrm{TP}+\mathrm{FP}) \times(\mathrm{TP}+\mathrm{FN}) \times$ $(\mathrm{TN}+\mathrm{FP}) \times(\mathrm{TN}+\mathrm{FN})$. The $F$-measure combines precision and recall into their harmonic mean, and is defined as $2 \times$ precision $\times$ recall $/($ precision + recall $)$, where precision is defined as $\mathrm{TP} /(\mathrm{TP}+\mathrm{FP})$ and measures the proportion of the positive pairs correctly predicted as interacting.

To evaluate the prediction performance of each AODE, a 10-fold cross validation (CV) was carried out. In the 10 -fold $\mathrm{CV}$, a data set was divided into 10 subsets, and each subset was used as a testing set and the remaining subsets were used as a training set. This process was repeated 10 times, and then the prediction performances were averaged over all the test results.

\section{Results}

In this section, we first assess critically the AODE models based on three homology-based features encoded in a single feature vector. We then demonstrate high predictive performance of our proposed method using a large, human PPI data set compiling recently identified interactions.

\section{Can proximity between homologous proteins in a PPI network contribute to predictions?}

We hypothesized that two proteins would have more potential to interact, if their homologous proteins exist in proximity of each other in a known PPI network. Such a proximal pair, even if not known to interact directly, may form a complex with other proximal proteins or reside in common subcellular locations, thereby increasing the chances of their homologues interacting directly. To confirm our hypothesis, we divided Dset1 into 10 subsets, treated each subset as a test set and constructed a PPI network from the remaining subsets. For each pair in the test set, we identified homologous protein pairs (with a BLAST e-value cut-off $\leq 10^{-3}$ ) and obtained the smallest SPW (a sum of edge weights along the shortest path; see METHODS) in the PPI network. In this study, an edge weight of 1.0 was used as a default weight value. This process was repeated 10 times, and the average number of protein pairs with a given SPW was counted.

Figure 5 shows the percentage of protein pairs with different SPWs. Note that, in this figure, an SPW of 0 means a known interaction of a homologous protein with itself and that of 1.0 means a known interaction between a homologous protein pair. Also, a homologous protein pair indirectly linked by $n$ proteins has an SPW of $n+1.0$. In consequence, the percentage of positive pairs with an SPW $\leq 1.0$ was about five times lager than that of negative pairs (39\% vs $7 \%$ ). That of positive pairs with an SPW of 2.0 (about 23.2\%) was about 10 percentage point lager than that of negative pairs (about 13.1\%). Furthermore, a large proportion of positive pairs had SPWs of $\leq 2.0$ (on average, $62.3 \%$ ), compared to a relatively small proportion of negative pairs (on average, 


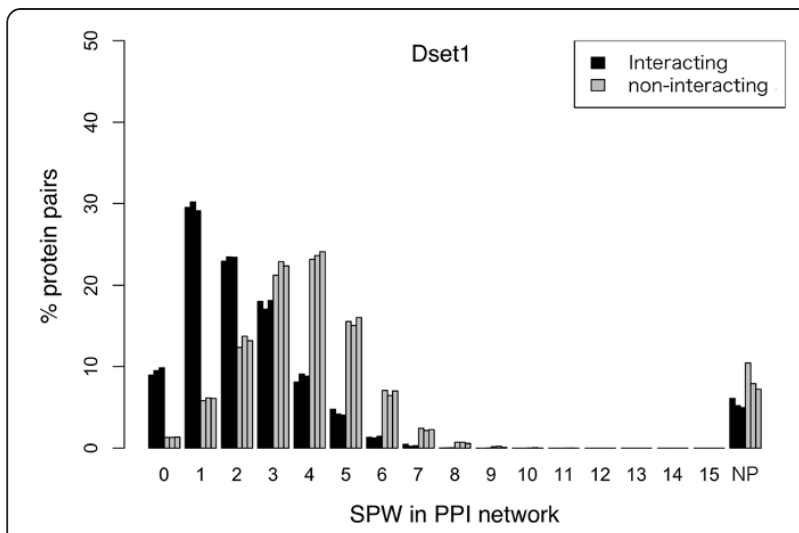

Figure $\mathbf{5}$ The percentage of protein pairs with different SPWs in the PPI network generated from Dset1. An SPW of 0 means a known interaction of a homologous protein with itself and that of 1.0 means a known interaction between a homologous protein pair. Also, a homologous protein pair indirectly linked by $n$ proteins has an SPW of $n+1.0$. NP (No Path) indicates that there was no path between two homologous proteins.

20.4\%). We, therefore, concluded that proximity between homologous proteins in a PPI network could contribute to a discrimination of positive and negative pairs in the PPI prediction, especially, in SPWs $\leq 2.0$. Then, the SPW was chosen as a feature for training the AODE and denoted by $\mathrm{F}_{\mathrm{Net}}$.

\section{Prediction performance of AODEs}

The AODE was trained and tested on Dset1, a highly imbalanced data set [29]. For a pair of proteins, three different homology-based features, $F_{\text {Seq }}, F_{\text {Dom }}$ and $F_{\text {Net }}$, were computed (see Methods). The order of two proteins to define $\mathrm{F}_{\text {Seq }}$ was determined by selecting its corresponding point in the half-space of the four-dimensional feature space. Then, a set of FVs was constructed to build the AODEs. In addition, all the feature values of a FV were discretized using the entropy-based discretization method [36]. (See Methods and Figures 1, 2, 3 and 4 for more details). In order to assess the predictability of each feature and create the best AODE model, all possible combinations of the three features were examined, where each AODE was evaluated in a 10 -fold $\mathrm{CV}$ on each independent set of Dset1. In each round of the CV, the AODE was tested on each subset of the independent set, i.e., about 500 positive pairs and 200,000 negative pairs, after trained on the remaining subsets, i.e., about 4,500 positive pairs and 1,800,000 negative pairs. Table 1 shows the prediction performances of different AODEs and, for comparison purposes, those of different NBCs, for different combinations of heterogeneous features. The probability model of the NBC has been introduced in our previous paper [40]. Furthermore, for reference, we included previously reported performances of four different methods benchmarked on Dset1 [29] (Additional file 1: Table S1).
While all these methods take protein sequences as input, a direct comparison of the performance is difficult, because our proposed method is based on heterogeneous input features, in contrast to the reported, purely sequencebased methods of M1 M4, which do not use homologous protein sequences explicitly (see Additional file 1: Table S1 for more details of these methods).

Of AODEs-I III based on a single feature, AODE-I achieved the highest pAUC $0.5 \%$ of $0.15(\mathrm{AUC}=0.69)$, and AODE-III achieved the highest AUC of 0.77 (pAUC $0.5 \%=$ 0.02 ). Of AODE-IV VI, which were created by integrating two features, AODE-VI (based on $F_{\text {Dom }}$ and $F_{N e t}$ ) achieved the highest AUC of 0.79. Although AODE-II (based on only $\mathrm{F}_{\mathrm{Dom}}$ ) gave the lowest $\mathrm{AUC}=0.57$ $\left(\mathrm{pAUC}_{0.5 \%}=0.07\right)$ of all three single-feature-based AODEs, integrating $F_{\text {Dom }}$ with $F_{\text {Seq }}$ or $F_{\text {Net }}$ improved both AUC and pAUC ${ }_{0.5 \%}$, as shown in AODE-IV (AUC = 0.71, $\left.\mathrm{pAUC}_{0.5 \%}=0.16\right)$ and AODE-VI (AUC = 0.79, $\left.\mathrm{pAUC}_{0.5 \%}=0.09\right)$. AODE-VII integrated all three features and it achieved the highest performance in this $\mathrm{CV}$, in terms of both AUC (0.79) and pAUC ${ }_{0.5 \%}(0.16)$. In comparison with NBC, AODEs-II and III were identical models to NBCs-II and III, respectively, and no performance difference was observed for methods I and IV. However, including a single dependency with $F_{\mathrm{Net}}$ achieved small but statistically significant improvements over NBC. (The p-values from the $t$-test (assuming equal variances) for comparison of AUC values of those methods, i.e., V, VI and VII, were all < 10e-7). While pAUC ${ }_{0.5 \%}$ values of AODE-VII and NBC-VII were comparable ( $p$-value = $0.603)$, pAUC values up to $\mathrm{FPR} \leq 10 \%$, i.e., pAUC $10 \%$, were 0.40 for AODE and 0.37 for $\mathrm{NBC}$, respectively ( $p$-value $=$ 6.4e-08). These results indicate that making a weaker dependence between features can contribute to the improvement of performance, also in the higher specificity range. Furthermore, AODE-VII outperformed all four previously reported methods in terms of AUC. (As mentioned above, the comparison should be taken with caution and note that pAUCs for M1 M4 were not given in [29]). ROC curves for AODE-VII on Dset1 are shown in Figure 6. In conclusion, AODE-VII achieved the highest performance on Dset1 and thus, it was selected as the best AODE and named PSOPIA (Prediction Server Of Proteinprotein InterActions).

\section{Evaluation of PSOPIA using an independent data set}

In order to evaluate our proposed method further, we compared PSOPIA (AODE-VII) with BIPS, a recently developed prediction server based on homologues of two interacting proteins [21]. Because BIPS is based on large, up-to-date PPI data, integrated from several PPI databases by using the BIANA software framework [24], it is considered to have advantages over other similar methods in retrieving homologous PPIs [18,22]. 
Table 1 Performances of AODEs and NBCs trained on Dset1

\begin{tabular}{|c|c|c|c|c|c|c|}
\hline \multirow{2}{*}{\multicolumn{2}{|c|}{$\begin{array}{c}\text { Method } \\
\text { Performance measure }\end{array}$}} & \multicolumn{2}{|c|}{ AODE } & \multicolumn{2}{|c|}{ NBC } & \multirow[t]{2}{*}{$p$-value } \\
\hline & & AUC & pAUC $_{0.5 \%}$ & AUC & pAUC $_{0.5 \%}$ & \\
\hline 1 & $F_{\text {Seq }}$ & $0.69 \pm 0.01$ & 0.15 & $0.69 \pm 0.01$ & 0.15 & 0.734 \\
\hline$\|$ & $F_{\text {Dom }}$ & $0.57 \pm 0.01$ & 0.07 & $0.57 \pm 0.01$ & 0.07 & 1.0 \\
\hline III & $\mathrm{F}_{\mathrm{Net}}$ & $0.77 \pm 0.01$ & 0.02 & $0.77 \pm 0.01$ & 0.02 & 1.0 \\
\hline IV & $\mathrm{F}_{\mathrm{Seq}}+\mathrm{F}_{\mathrm{Dom}}$ & $0.71 \pm 0.01$ & 0.16 & $0.70 \pm 0.01$ & 0.16 & 0.077 \\
\hline V & $\mathrm{F}_{\mathrm{Seq}}+\mathrm{F}_{\mathrm{Net}}$ & $0.79 \pm 0.01$ & 0.15 & $0.77 \pm 0.01$ & 0.15 & $2.8 \mathrm{e}-08$ \\
\hline VI & $\mathrm{F}_{\mathrm{Dom}}+\mathrm{F}_{\mathrm{Net}}$ & $0.79 \pm 0.01$ & 0.09 & $0.77 \pm 0.01$ & 0.09 & $2.7 e-08$ \\
\hline VII & $\mathrm{F}_{\mathrm{Seq}}+\mathrm{F}_{\mathrm{Dom}}+\mathrm{F}_{\mathrm{Net}}$ & $0.79 \pm 0.01$ & 0.16 & $0.77 \pm 0.01$ & 0.16 & $3.9 e-14$ \\
\hline
\end{tabular}

The AUC and the PAUC $_{0.5 \%}$ values calculated with 10 -fold CV on Dset1 are shown. AUC values given are the mean \pm standard deviation. P-values are calculated.

In addition, BIPS can use heterogeneous information similar to PSOPIA for filtering out prediction results, such as information about domain-domain interactions (DDIs) in iPfam [41] and 3DID [42] and annotations from UniProt [32] and GO [43], as well as BLAST-based sequence similarities to a known interacting protein pair. For these reasons, we evaluated the predictability of both PSOPIA and BIPS on Dset2, a data set, which was compiled from a recent release of the BioGrid database and which included only the PPIs identified after BIPS was developed and Dset1 was created (see Methods).

PSOPIA was retrained on the whole of Dset1 and a sequence database used for BLAST was formatted with all the sequences in Dset1. A threshold value of 0.293 was chosen, because it gave the highest $F$-measure $(0.160)$ in the 10 -fold CV on Dset1 (recall $=15.5 \%$, precision $=17.0 \%$, specificity $=99.8 \%$, MCC $=0.160$ ). For BIPS, since we were unable to optimize the parameters, we used the default values by the web server: joint identities (the geometric mean of individual BLAST sequence identities) $\geq 80 \%$, joint e-values (the geometric mean of individual BLAST evalues) $\geq 1.0 \times \mathrm{e}^{-10}$ and template sequence coverage $\geq 80 \%$ (see [21] for more details of these parameters). In addition to the default "filter by template interactions", we also examined two additional filtering conditions: information about DDIs in iPfam or 3DID, and GO annotations (biological process, cellular component or molecular function). The BIPS server accepts sequences of interest or a list of protein identifiers, evaluates potential interactions between all possible sequence pairs and reports only likely (high-scoring) interactions. Therefore, we submitted all the unique sequences in Dset2 to the BIPS server, retrieved the results and defined all the reported pairs to be positive predictions (interacting) and all non-

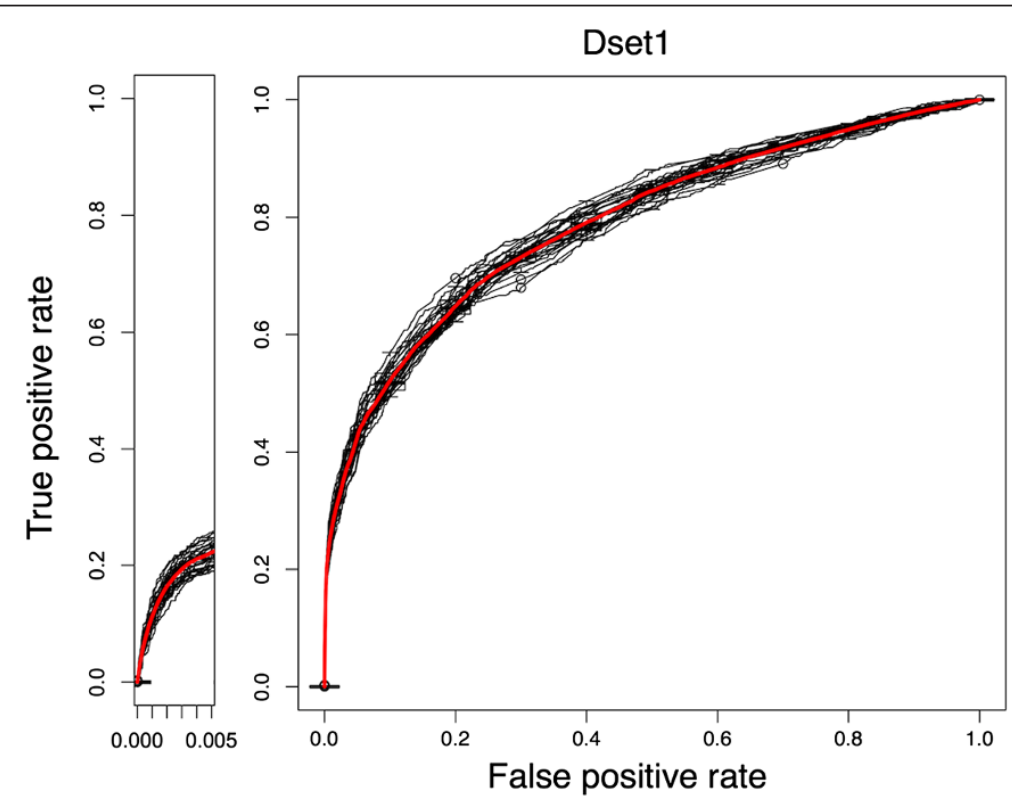

Figure 6 ROC curves for the AODEs trained with all three features in 10- fold CV. The ROC curves for the AODE-VII are shown, as well as the averaged ROC curve (in red). This model achieved an AUC of 0.79 and a PAUC $C_{0.5 \%}$ of $0.16 \%$ on average, in each round of $10-$ fold $C V$ on each of the three independent data sets in Dset1. 
reported pairs to be negative predictions (non-interacting). If a positively predicted pair was found in either the positive or the negative set of Dset2, it was regarded as a true positive or a false positive, respectively. If a negatively predicted pair was found in either the positive or the negative set of Dset2, it was regarded as a false negative or a true negative, respectively. All the other predicted interactions were ignored. In this comparison, we aimed to evaluate the true predictability of these methods, i.e., whether they can predict novel PPIs that have never been observed before, not the data search capability to identify already known PPIs in a database. Thus, we excluded from the evaluation any protein pair $\left(S_{A}, S_{B}\right)$ if either BIPS or PSOPIA detected a known interacting protein pair $\left(T_{A}\right.$, $T_{B}$ ) in their database (with BLAST e-values of 0 for $S_{A \text { - }}$ $T_{A}$ and $\left.S_{B}-T_{B}\right)$.

Table 2 shows the prediction performances of PSOPIA and BIPS on Dset2. BIPS predictions using template interactions from only human PPIs (taxonomy ID $=9609$ ) (I-A) achieved an $F$-measure of 0.009 (recall $=0.51 \%$, precision $=2.72 \%$ ). Adding additional information about DDIs and GO annotations reduced the false positives but also reduced the true positives and did not improve the prediction performance (II, III). Furthermore, the use of template interactions from all species increased the false positive in all three options (I) (III) of the BIPS predictions. On the other hand, PSOPIA achieved a higher $F$-measure of 0.030 (recall $=3.33 \%$, precision $=$ $2.77 \%$ ) at the chosen threshold of 0.293 . In addition, by raising the threshold to 0.67 to obtain the recall value of $0.5 \sim 0.6 \%$ (comparable to that of BIPS), PSOPIA achieved much higher precision (13.71\%) than BIPS (2.72\%). In conclusion, in the benchmarking on Dset2, PSOPIA demonstrated higher predictability than BIPS in terms of the F-measure.

\section{Discussion}

We have proposed a new AODE-based method for predicting PPIs based on known homologous PPIs by using three different features, $F_{S e q}, F_{\text {Dom }}$ and $F_{N e t}$. In constructing Dset1 [29] used for training and testing the AODEs, randomly sampled protein pairs that had not been known to interact with each other were used as a negative data set, because of the limited availability of high-quality negative PPI data, either manually curated or experimentally determined (for example, only 1,892 negative PPIs constructed with 1,257 proteins in the negatome database [44]). In reality the number of negative PPIs should be much larger than that of positive PPIs [29,31] and therefore, we trained and evaluated the AODEs on a data set with a large number of negative data. The AODEs were able to deal with this large and imbalanced PPI dataset effectively and they were easily trained within several CPU minutes.

In order to deal with symmetry in the protein order and allow the concatenation of a set of features for individual proteins in a FV, several kernels have been developed in sequence-based methods using a support vector machine (SVM) $[6,7,10]$. In this study, we proposed a simple geometric selection of FVs in a half space of the symmetrical FV space. Although no comparison can be made between these two approaches, our FV selection method is simple and can be incorporated in any ML method.

Table 2 Evaluation of true prediction performance on Dset2

\begin{tabular}{|c|c|c|c|c|c|c|c|c|}
\hline Method & TP & FP & TN & FN & Sp (\%) & $\operatorname{Re}(\%)$ & $\operatorname{Pr}(\%)$ & $\mathbf{F}$ \\
\hline PSOPIA $(\theta=0.293$, the higheset $F)$ & 143 & 5,026 & $1,766,423$ & 4,152 & 99.72 & 3.33 & 2.77 & 0.030 \\
\hline PSOPIA $(\theta=0.670)$ & 24 & 151 & $1,771,298$ & 4,271 & 99.99 & 0.56 & 13.71 & 0.012 \\
\hline PSOPIA $(\theta=0.890)$ & 4 & 31 & $1,771,418$ & 4,291 & 99.99 & 0.09 & 11.43 & 0.002 \\
\hline \multicolumn{9}{|c|}{ (I) BIPS, only filtered by the template interactions } \\
\hline (A) Template: Taxonomy ID = 9609 (human) & 19 & 680 & $1,765,404$ & 3,710 & 99.96 & 0.51 & 2.72 & 0.009 \\
\hline (B) Template: all species & 19 & 833 & $1,765,005$ & 3,705 & 99.95 & 0.51 & 2.23 & 0.008 \\
\hline \multicolumn{9}{|c|}{ (II) BIPS, filtered by known DDIs (iPfam or 3DID) } \\
\hline (A) Template: Taxonomy ID = 9609 (human) & 5 & 60 & $1,771,096$ & 4,261 & 99.99 & 0.12 & 7,69 & 0.002 \\
\hline (B) Template: all species & 5 & 72 & $1,771,059$ & 4,256 & 99.99 & 0.12 & 6.49 & 0.002 \\
\hline
\end{tabular}

(III) BIPS, filtered by known DDIs (iPfam or 3DID) and GO; biological process, cellular component or molecular function

$\begin{array}{llllllll}\text { (A) Template: Taxonomy ID = 9609 (human) } & 3 & 47 & 1,771,245 & 4,284 & 99.99 & 0.07 & 0.001 \\ \text { (B) Template: all species } & 3 & 56 & 1,771,216 & 4,280 & 99.99 & 0.07 & 5.08\end{array}$

For PSOPIA trained on Dset1 (a data set independent of Dset2), the best threshold value, 0.995, which gave the highest F-measure in the 10-fold CV, was used to classify a pair of proteins as interacting or non-interacting. For BIPS, the default values in homologue conditions were used: joint identities $\geq 80 \%$, joint e-values $\geq 1.0 \times \mathrm{e}^{-10}$, and template sequence coverage $\geq 80 \%$ (see [21] for more details of these parameters). In addition to the filtering by the template interactions only (I), two additional filters were applied: (II) filtered by known DDIs in iPfam or 3DID and (III) filtered by known DDIs and GO annotations (biological process, cellular component or molecular function). Furthermore, two template interactions, (A) only from human (taxonomy ID $=9609)$ and $(B)$ from all species, were also considered. 
The predictability of the AODEs, which include a single dependency between the features, was illustrated in a 10fold CV on Dset1, and then the AODE trained using all three features, named PSOPIA, achieved the highest performance in terms of both $\mathrm{AUC}(0.79)$ and $\mathrm{pAUC}_{0.5 \%}$ (0.16). In comparison with the NBC, which assumes conditional independence of all three features, PSOPIA improved AUC by 0.02 (p-value $<2.8 \mathrm{e}-08$ ) and $\mathrm{pAUC}_{10 \%}$ by 0.03 ( $p$-value $=6.4 \mathrm{e}-08)$. We further tested PSOPIA on Dset2, an independent data set, and compared its performance with that of BIPS, a recently reported homology-based method. By excluding the identification of interacting protein pairs already in the database, PSOPIA (threshold= 0.670 ) achieved higher precision of $13.71 \%$ than that of BIPS $(2.72 \%)$ at a recall level of $0.5 \sim 0.6 \%$, and thus demonstrating higher predictability than BIPS in terms of the $F$ measure. The $F$-measure is generally known as a useful and reliable measure to evaluate different methods that have different trade-off relations between precision and recall.

Further improvements of PSOPIA may be possible by creating a large up-to-date PPI dataset integrated from several databases, because a larger PPI database provides a better chance of detecting known PPIs homologous to a target protein pair. It is still unclear, however, whether we should include cross-species data in such a database. In this study, we evaluated BIPS on Dset 2 and showed that the use of interactions from different species did not reduce the false positives. Also, Park [31] and Pitre et al. [45] investigated whether interactions for a pair of proteins in a target species can be predicted using a method trained on known PPI data from different species and observed no significant improvements in the performance of the predictors. Thus, it remains to be seen whether the AODE, a probability-based ML method, can improve the prediction performance using interactions from different species as a training dataset. Moreover, it will be worth attempting to change edge weights in a PPI network and distinguish the interaction type, for example, using numerical parameters given by Kerrien et al. [46] or similarities in GO annotations [43].

\section{Conclusions}

In this study, we have illustrated that proximity in a known PPI network between two proteins homologous to a target protein pair contributes to the prediction of whether the target proteins interact or not. Then, we have applied this feature $\mathrm{F}_{\mathrm{Net}}$ to the PPI prediction with two other features, $F_{\text {Seq }}$ and $F_{\text {Dom. }}$. Our best AODE, which achieved an AUC of $0.79\left(\mathrm{pAUC}_{0.5 \%}=0.16\right)$ in a 10 -fold CV on a highly imbalanced data set, will hopefully contribute to the identification of novel PPIs and the estimation of complete PPI networks. The method proposed in this study is freely available on the web at
http://mizuguchilab.org/PSOPIA, and Dset2 used for the evaluation can be downloaded from the same URL.

\section{Additional file}

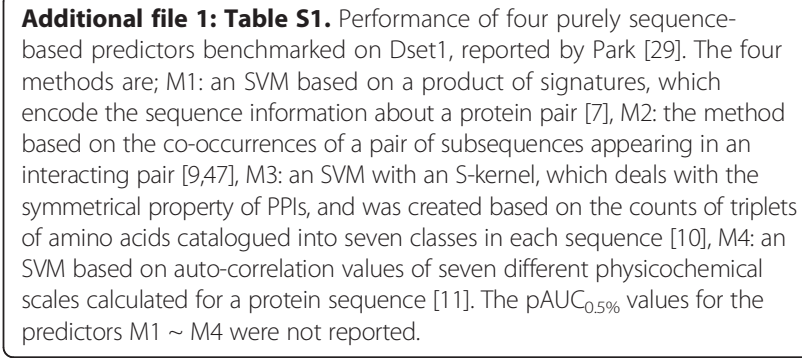

\section{Competing interests}

The authors declare that they have not competing interests.

\section{Authors' contributions}

YM developed the methodology and the web server, performed the data analysis and wrote the paper. KM contributed to designing the research, the development and the writing of the manuscript. All authors read and approved the final manuscript.

\section{Acknowledgements}

This work was supported by Platform for Drug Discovery, Informatics, and Structural Life Science from the Ministry of Education, Culture, Sports, Science and Technology, Japan. Furthermore, this study was also in part supported by the Industrial Technology Research Grant Program in 2007 (Grant Number 07C46056a) from New Energy and Industrial Technology Development Organization (NEDO) of Japan, and also by Grants-in-Aid for Scientific Research from the Ministry of Education, Culture, Sports, Science, and Technology (Grant Numbers 25430186 and 25293079) and from the Ministry of Health, Labor, and Welfare ("The Adjuvant database project") to K.M. We thank Shandar Ahmad for carefully reading the manuscript and for helpful comments.

Received: 30 January 2014 Accepted: 17 June 2014

Published: 23 June 2014

\section{References}

1. von Mering C, Krause R, Snel B, Cornell M, Oliver SG, Fields S, Bork P: Comparative assessment of large-scale data sets of protein-protein interactions. Nature 2002, 417(6887):399-403.

2. Han JD, Dupuy D, Bertin N, Cusick ME, Vidal M: Effect of sampling on topology predictions of protein-protein interaction networks. Nat Biotechnol 2005, 23(7):839-844.

3. Bock JR, Gough DA: Predicting protein-protein interactions from primary structure. Bioinformatics 2001, 17(5):455-460.

4. Sprinzak E, Margalit $\mathrm{H}$ : Correlated sequence-signatures as markers of protein-protein interaction. J Mol Biol 2001, 311(4):681-692.

5. Gomez SM, Noble WS, Rzhetsky A: Learning to predict protein-protein interactions from protein sequences. Bioinformatics 2003, 19(15):1875-1881.

6. Ben-Hur A, Noble WS: Kernel methods for predicting protein-protein interactions. Bioinformatics 2005, 21(Suppl 1):i38-46.

7. Martin S, Roe D, Faulon JL: Predicting protein-protein interactions using signature products. Bioinformatics 2005, 21(2):218-226.

8. Nanni L, Lumini A: An ensemble of K-local hyperplanes for predicting protein-protein interactions. Bioinformatics 2006, 22(10):1207-1210.

9. Pitre S, Dehne F, Chan A, Cheetham J, Duong A, Emili A, Gebbia M, Greenblatt J, Jessulat M, Krogan N, Luo X, Golshani A: PIPE: a proteinprotein interaction prediction engine based on the re-occurring short polypeptide sequences between known interacting protein pairs. BMC Bioinformatics 2006, 7:365.

10. Shen J, Zhang J, Luo X, Zhu W, Yu K, Chen K, Li Y, Jiang H: Predicting protein-protein interactions based only on sequences information. Proc Natl Acad Sci U S A 2007, 104(11):4337-4341. 
11. Guo Y, Yu L, Wen Z, Li M: Using support vector machine combined with auto covariance to predict protein-protein interactions from protein sequences. Nucleic Acids Res 2008, 36(9):3025-3030.

12. Roy S, Martinez D, Platero H, Lane T, Werner-Washburne M: Exploiting amino acid composition for predicting protein-protein interactions. PLoS One 2009, 4(11):e7813.

13. Yu CY, Chou LC, Chang DT: Predicting protein-protein interactions in unbalanced data using the primary structure of proteins. BMC Bioinformatics 2010, 11:167.

14. Yu J, Guo M, Needham CJ, Huang Y, Cai L, Westhead DR: Simple sequencebased kernels do not predict protein-protein interactions. Bioinformatics 2010, 26(20):2610-2614.

15. Guo Y, Li M, PU X, Li G, Guang X, Xiong W, Li J: PRED_PPI: a server for predicting protein-protein interactions based on sequence data with probability assignment. BMC Res Notes 2010, 3:145.

16. Deng M, Mehta S, Sun F, Chen T: Inferring domain-domain interactions from protein-protein interactions. Genome Res 2002, 12(10):1540-1548.

17. Hayashida M, Kamada M, Song J, Akutsu T: Conditional random field approach to prediction of protein-protein interactions using domain information. BMC Syst Biol 2011, 5(Suppl 1):S8

18. Chen CC, Lin CY, Lo YS, Yang JM: PPISearch: a web server for searching homologous protein-protein interactions across multiple species. Nucleic Acids Res 2009, 37(Web Server issue):W369-375.

19. Matthews LR, Vaglio P, Reboul J, Ge H, Davis BP, Garrels J, Vincent S, Vidal $M$ : Identification of potential interaction networks using sequence-based searches for conserved protein-protein interactions or "interologs". Genome Res 2001, 11(12):2120-2126

20. Yu H, Luscombe NM, Lu HX, Zhu X, Xia Y, Han JD, Bertin N, Chung S, Vidal $M$, Gerstein M: Annotation transfer between genomes: protein-protein interologs and protein-DNA regulogs. Genome Res 2004, 14(6):1107-1118.

21. Garcia-Garcia J, Schleker S, Klein-Seetharaman J, Oliva B: BIPS: BIANA Interolog Prediction Server. A tool for protein-protein interaction inference. Nucleic Acids Res 2012, 40(Web Server issue):W147-151.

22. Wiles AM, Doderer M, Ruan J, Gu TT, Ravi D, Blackman B, Bishop AJ: Building and analyzing protein interactome networks by cross-species comparisons. BMC Syst Biol 2010, 4:36

23. Gallone G, Simpson Tl, Armstrong JD, Jarman AP: Bio:Homology: InterologWalk-a Perl module to build putative protein-protein interaction networks through interolog mapping. BMC Bioinformatics 2011, 12:289.

24. Garcia-Garcia J, Guney E, Aragues R, Planas-Iglesias J, Oliva B: Biana: a software framework for compiling biological interactions and analyzing networks. BMC Bioinformatics 2010, 11:56.

25. Webb Gl, Boughton JR, Wang Z: Not so naive Bayes: Aggregating one-dependence estimators. In Machine Learning. Volume 58, Issue 1. Netherlands: Springer; 2005:5-24.

26. Garcia-Jimenez B, Juan D, Ezkurdia I, Andres-Leon E, Valencia A: Inference of functional relations in predicted protein networks with a machine learning approach. PLoS One 2010, 5(4):e9969.

27. Webb Gl, Boughton JR, Zheng F, Ting KM, Salem H: Learning by extrapolation from marginal to full-multivariate probability distributions: decreasingly naive Bayesian classification. In Machine Learning. Volume 86, Issue 2. Netherlands: Springer; 2012:233-272.

28. Yu H, Paccanaro A, Trifonov V, Gerstein M: Predicting interactions in protein networks by completing defective cliques. Bioinformatics 2006, 22(7):823-829.

29. Park $Y$, Marcotte EM: Revisiting the negative example sampling problem for predicting protein-protein interactions. Bioinformatics 2011 27(21):3024-3028.

30. Stark C, Breitkreutz BJ, Reguly T, Boucher L, Breitkreutz A, Tyers M: BioGRID: a general repository for interaction datasets. Nucleic Acids Res 2006, 34(Database issue):D535-539.

31. Park Y: Critical assessment of sequence-based protein-protein interaction prediction methods that do not require homologous protein sequences. BMC Bioinformatics 2009, 10:419.

32. UniProt C: Reorganizing the protein space at the Universal Protein Resource (UniProt). Nucleic Acids Res 2012, 40(Database issue):D71-75

33. Altschul SF, Gish W, Miller W, Myers EW, Lipman DJ: Basic local alignment search tool. J Mol Biol 1990, 215(3):403-410.

34. Henikoff $S$, Henikoff JG: Amino acid substitution matrices from protein blocks. Proc Natl Acad Sci U S A 1992, 89(22):10915-10919.
35. Punta M, Coggill PC, Eberhardt RY, Mistry J, Tate J, Boursnell C, Pang N, Forslund K, Ceric G, Clements J, Heger A, Holm L, Sonnhammer EL, Eddy SR, Bateman A, Finn RD: The Pfam protein families database. Nucleic Acids Res 2012, 40(Database issue):D290-301.

36. Fayyad UM, Rani KB: Multi-interval discretization of continuous-valued attributes for classification learning. In Proceedings of the International Joint Conference on Uncertainty in Al (Q334 1571 1993). 1993:1022-1027.

37. Fawcett T: An introduction to ROC analysis. Pattern Recognition Lett 2006, 27(8):861-874

38. Matthews BW: Comparison of the predicted and observed secondary structure of T4 phage lysozyme. Biochim Biophys Acta 1975, 405(2):442-451.

39. Hripcsak G, Rothschild AS: Agreement, the f-measure, and reliability in information retrieval. J Am Med Inform Assoc 2005, 12(3):296-298.

40. Murakami Y, Mizuguchi K: Applying the Naive Bayes classifier with kernel density estimation to the prediction of protein-protein interaction sites. Bioinformatics 2010, 26(15):1841-1848.

41. Finn RD, Marshall M, Bateman A: iPfam: visualization of protein-protein interactions in PDB at domain and amino acid resolutions. Bioinformatics 2005, 21(3):410-412

42. Stein A, Ceol A, Aloy P: 3did: identification and classification of domainbased interactions of known three-dimensional structure. Nucleic Acids Res 2011, 39(Database issue):D718-723.

43. Ashburner M, Ball CA, Blake JA, Botstein D, Butler H, Cherry JM, Davis AP, Dolinski K, Dwight SS, Eppig JT, Harris MA, Hill DP, Issel-Tarver L, Kasarskis A, Lewis S, Matese JC, Richardson JE, Ringwald M, Rubin GM, Sherlock G: Gene ontology: tool for the unification of biology. The Gene ontology consortium. Nat Genet 2000, 25(1):25-29.

44. Smialowski $P$, Pagel $P$, Wong $P$, Brauner B, Dunger I, Fobo G, Frishman G, Montrone C, Rattei T, Frishman D, Ruepp A: The Negatome database: a reference set of non-interacting protein pairs. Nucleic Acids Res 2010, 38(Database issue):D540-544

45. Pitre $S$, Hooshyar M, Schoenrock A, Samanfar B, Jessulat M, Green JR Dehne F, Golshani A: Short Co-occurring Polypeptide Regions Can Predict Global Protein Interaction Maps. Sci Rep 2012, 2:239.

46. Kerrien S, Aranda B, Breuza L, Bridge A, Broackes-Carter F, Chen C, Duesbury M, Dumousseau M, Feuermann M, Hinz U, Jandrasits C, Jimenez RC, Khadake J, Mahadevan U, Masson P, Pedruzzi I, Pfeiffenberger E, Porras P, Raghunath A, Roechert B, Orchard S, Hermjakob H: The IntAct molecular interaction database in 2012. Nucleic Acids Res 2012, 40(Database issue):D841-846.

47. Pitre S, North C, Alamgir M, Jessulat M, Chan A, Luo X, Green JR, Dumontier M, Dehne F, Golshani A: Global investigation of protein-protein interactions in yeast Saccharomyces cerevisiae using re-occurring short polypeptide sequences. Nucleic Acids Res 2008, 36(13):4286-4294

doi:10.1186/1471-2105-15-213

Cite this article as: Murakami and Mizuguchi: Homology-based prediction of interactions between proteins using Averaged One-Dependence Estimators. BMC Bioinformatics 2014 15:213.

\section{Submit your next manuscript to BioMed Central and take full advantage of:}

- Convenient online submission

- Thorough peer review

- No space constraints or color figure charges

- Immediate publication on acceptance

- Inclusion in PubMed, CAS, Scopus and Google Scholar

- Research which is freely available for redistribution 